\title{
Role of Colour Doppler in the Prediction of Perinatal Outcome in Intra- uterine Growth Restrictions
}

\author{
Authors \\ Kalyani Parida ${ }^{1}$, Dvijottam Biswal ${ }^{2}$ \\ ${ }^{1}$ Utkal University, S.C.B. Medical College \& Hospital, Cuttack, Odisha, India \\ ${ }^{2}$ S.O.A.University, IMS \& SUM Hospital, Bhubaneswar, Odisha, India
}

\begin{abstract}
Analyzing umbilical artery, middle cerebral artery, and uterine artery by Doppler Velocimetry in intrauterine growth restricted foetuses, in order to predict adverse peri-natal outcomes and also the clinical management of such pregnancies. A prospective study with 60 ante-natal women in their third trimester was included in the study and was subjected to colour Doppler examination. Findings of Doppler studies were used to evaluate foetal well-being and were correlated with perinatal outcome. Out of 60 foetuses, 38 had at least one unfavourable perinatal outcome, including six intrauterine deaths, admission of 12 neonates to NICU, low APGAR score in 14 neonates, and emergency caesarian delivery of 18 babies. A single cut off value (1.08) for the ratio of middle cerebral artery and umbilical artery pulsatility index was used and the value above 1.08 was considered normal and below it is abnormal. The value of cerebro-umbilical ratio revealed that $63.3 \%$ of foetuses had redistribution of blood to the brain. The present study showed that in women with intrauterine growth restricted foetuses colour Doppler ultrasound is a useful tool to evaluate the neonatal outcomes. The cerebro-umbilical ratio was a better predictor for foetal outcome in terms of sensitivity and predictive value. Moreover, the absent/reversal of end diastolic flow in umbilical artery represents altered blood flow and indicates the risk of chronic hypoxia in foetus leading to high mortality.
\end{abstract}

Keywords- Intra-uterine growth restriction, antenatal women, cerebro-umbilical ratio, chronic hypoxia.

\section{Introduction}

Intra-uterine growth restriction (IUGR) is defined as pathologic decrease in foetal growth rate with sonographic estimated foetal weight less than 10th percentile for its gestational age ${ }^{[1]}$. IUGR is considered to be one of the most common and complex problems in modern obstetrics according to the American College of Obstetricians and Gynaecologists ${ }^{[1]}$. The estimated occurrence of IUGR among healthy and well-nourished mother is around $3-5 \%$, whereas the occurrence is $15-20 \%$ more in women with hypertension or previous growth restricted foetus ${ }^{[2]}$. The incidence of IUGR differs among populations, races and countries ${ }^{[3]}$. Normally, IUGR results from various maternal, foetal, or placental factors, and even a combination of any of these factors ${ }^{[3]}$. Maternal factors include chronic hypertension, renal disease, collagen vascular disease, pre-gestational diabetes, malnutrition, and drug or alcohol abuse leading to placental insufficiency. Foetal etiologies causing IUGR include chromosomal aberrations like, trisomies of chromosomes 13,18,21,16, malformations, inborn error of metabolism, perinatal viral or protozoan infections, and preterm birth ${ }^{[4]}$. Anatomical, chromosomal, morphological 
abnormalities as well as placental infections have been implicated as placental factors responsible for IUGR ${ }^{[3]}$. Better understanding of these etiological conditions could result in improved prediction, prevention, and management of foetal growth restriction.

IUGR foetuses showed eight to tenfold increase in perinatal mortality and $50-75 \%$ morbidity ${ }^{[5]}$. It is associated with an increased risk of adverse perinatal outcomes such as: still birth, intrapartum foetal acidosis, perinatal asphyxia, hypoglycemia, hypoxic ischemia encephalopathy ${ }^{[6]}$, and also reduced intellectual development and diseases like hypertension and obesity in adulthood ${ }^{[1]}$.

Accurate antenatal diagnosis of IUGR is one of the most important factors for improving perinatal consequence ${ }^{[7]}$. However, clinical management of IUGR, which includes appropriate identification of the truly growth-restricted foetus, selection of correct foetal surveillance and optimization of the delivery time, faces some major challenges at present ${ }^{1}$.

Doppler ultrasonography, a vital obstetric diagnostic tool for last 30 years ${ }^{[8]}$ is believed to be an efficient method of IUGR monitoring ${ }^{[6]}$. It is used to assess foetal, placental, utero placental, and foetoplacental blood circulations ${ }^{[8]}$. Doppler ultrasound of the uteroplacental and foeto-placental circulation provides information on the placental resistance and that of foetal circulation detects foetal response towards hypoxia. Umbilical arterial (Umb A) Doppler velocimetry is the most thoroughly estimated noninvasive test ${ }^{[9]}$ for accurate measurement of volume and velocity of foetoplacental blood flow. It helps in investigating foetal well-being ${ }^{[9]}$, predicting adverse pregnancy outcome, and monitoring foetal delivery time in high risk pregnancies. In fact, abnormality in Umb A waveform indicates a foetal compromise ${ }^{[8]}$ and subsequently considered to be a marker for uteroplacental insufficiency. Umb A absent end diastolic flow indicates high risk of foetal hypoxia due to altered blood velocity and reverse end diastolic flow represents acidotic foetuses ${ }^{[10]}$.

Prolonged hypoxic stress leads to circulatory adaptation in foetus resulting in redistribution of cardiac output, in order to supply oxygen constantly to the brain and heart ${ }^{[10]}$. The foetal hypoxia is also associated with increased diastolic flow in middle cerebral artery (MCA), which usually shows low diastolic flow, hence acts as a marker of vasodilation [11]. This increased diastolic flow reduces the MCA pulsatility index (PI) ${ }^{[10]}$. In fact, a series of Doppler ultrasound in IUGR foetuses showed a progressive reduction of MCA PI ${ }^{[12]}$. Moreover, percutaneous umbilical blood sampling revealed a significant correlation between mild arterial hypoxemia in IUGR foetuses and abnormal MCA PI ${ }^{[10]}$. Besides, uterine artery (UA) Doppler waveform analysis helps to differentiate between the placental causes of growth restriction from other causes ${ }^{[10]}$. A prospective study by Zimmerman et al revealed the importance of UA Doppler for predicting IUGR development ${ }^{[11]}$. Hence, PI of MCA and UA is considered to be valuable Doppler index for predicting pregnancy complications associated with IUGR ${ }^{[10]}$.

The aims and objectives of the present study were to evaluate the role of UA, Umb A, and MCA colour Doppler ultrasound in predicting adverse perinatal outcomes in clinically suspected IUGR pregnancies and also to determine the utility of Doppler velocimetry in the clinical management of IUGR pregnancies.

\section{Materials and Methods}

The study was conducted in the Department of Radio-diagnosis in S.C.B.Medical College \& Hospital, Cuttack. The study was performed on a sample of 60 antenatal women at their third trimester, diagnosed with IUGR foetus, based on grey scale ultrasound findings and referred for obstetric Doppler ultrasound in S.C.B.Medical College \& Hospital.

Inclusion criteria in the study were singleton pregnancy with gestational age more than 28 weeks and women with accurate dating of pregnancy established by an early first trimester ultrasound examination using CRL or BOD or with known LMP.

Exclusion criteria included: antenatal women with only clinically suspected IUGR without any grey 
scale ultrasound assessment and the women with history of rupture of membranes, active labour, multiple pregnancies, and foetuses with congenital anomalies.

The study protocol was approved by the hospital institutional review board and patients provided their written informed consent. Antenatal examinations included prenatal ultrasonography, and Doppler velocimetry of UA, Umb A, and MCA. Ultrasonography was performed using a PHILIPS HD7, ultrasound machine equipped with a 2.5-5 $\mathrm{MHz}$ convex electronic transducer. Doppler recordings were conducted in all cases following a detailed clinical history and US biometry. Follow up Doppler studies were performed to determine a favorable or a worsening trend in the Doppler indices, although, results of the first Doppler ultrasound were used for perinatal outcome analysis. The adverse perinatal outcome measures included low birth weight, perinatal death, emergency Caesarean section for foetal distress, low APGAR score (5 min APGAR score less than 7), and admission to neonatal intensive care unit (NICU) for complications of low birth weight. The pregnancy outcome was considered to be "adverse", if any one of the above mentioned perinatal complications were present, whereas the pregnancy outcome was considered to be "uneventful or favourable" when the above complications were absent.

Doppler velocimetry of UA, MCA, and Umb A was performed in each pregnant woman to predict pregnancy outcomes and colour Doppler images were used to improve the study results.

The UA resistance index $(\mathrm{RI})>0.58$ and presence of persistent UA early diastolic notch were considered as pathological Doppler signs ${ }^{[11]}$ which predict unfavorable perinatal outcomes.

Furthermore, value of Umb A and MCA PI recorded by Doppler velocimetry was considered to be abnormal if above 95th or below 5th percentiles respectively for gestational age, according to the reference values of Gramellini et al ${ }^{[13]}$. The MCA/Umb A PI ratio (cerebroumbilical ratio) is usually constant during the last 10 weeks of gestation, as reported by Wladimiroff et al ${ }^{[14]}$.
According to Cassady, $1971^{[15]}$, the statistical comparison of MCA PI/Umb A PI values showed no significant differences after 26 weeks of gestation. Since, the previous findings reported that the cerebroumbilical Doppler ratio remains constant between 30 to 40 weeks of gestation; a single cut off value could be used after 30th week. In the current study, the ratio of MCA PI/ Umb A PI was calculated and a single cutoff value of 1.08 for MCA/Umb A PI was fixed according to the reference values provided by Gramellini et al ${ }^{[13]}$, above which velocimetry was considered normal and below which it was considered abnormal.

\section{Statistical Methods}

Quantitative analyses were used to determine the values of Umb A PI, MCA PI and MCA/Umb A PI in relation to perinatal outcome. The sensitivity, specificity, positive predictive value, negative predictive value and diagnostic accuracy were determined for all using different statistical formulae (Tables 1 and 2).

Table. 1 Formulae used to determine different parameters for all Doppler measurements

\begin{tabular}{|l|c|}
\hline $\begin{array}{l}\text { Parameters determined for } \\
\text { all Doppler measurements }\end{array}$ & $\begin{array}{c}\text { Formulae used for } \\
\text { determination of the } \\
\text { parameters }\end{array}$ \\
\hline 1. Sensitivity & $\mathrm{A} /(\mathrm{A}+\mathrm{C}) \times 100$ \\
\hline 2. Specificity & $\mathrm{D} /(\mathrm{B}+\mathrm{D}) \times 100$ \\
\hline 3. Positive Predictive Value & $\mathrm{A} /(\mathrm{A}+\mathrm{B}) \times 100$ \\
\hline 4. Negative Predictive Value & $\mathrm{D} /(\mathrm{C}+\mathrm{D}) \times 100$ \\
\hline 5. Diagnostic Accuracy & $(\mathrm{A}+\mathrm{D}) /$ Total number of cases \\
\hline
\end{tabular}

Table. 2 Quantitative Analysis

\begin{tabular}{|l|c|c|c|}
\hline \multirow{2}{*}{ Test } & \multicolumn{3}{|c|}{ Criteria } \\
\cline { 2 - 4 } & Adverse & Uneventful & Total \\
\hline+ & $\mathrm{A}(\mathrm{TP})$ & $\mathrm{B}(\mathrm{FP})$ & $\mathrm{A}+\mathrm{B}$ \\
\hline- & $\mathrm{C}(\mathrm{FN})$ & $\mathrm{D}(\mathrm{TN})$ & $\mathrm{C}+\mathrm{D}$ \\
\hline Total & $\mathrm{A}+\mathrm{C}$ & $\mathrm{B}+\mathrm{D}$ & $\mathrm{N}$ \\
\hline
\end{tabular}

\section{RESULTS}

A prospective study of 60 antenatal patients was done after considering the inclusion and exclusion criteria. The age of the patients ranged from 19 to 31 years, with majority in age group 20-30 years. The average age was 24.5 years and least number of patients was more than 30 years of age (Table 3 ). Doppler Velocimetry of uterine, umbilical, and 
middle cerebral arteries was performed. Figure 1 shows normal and abnormal Doppler waveforms in different blood vessels.

Table. 3 Distribution of age of the patients

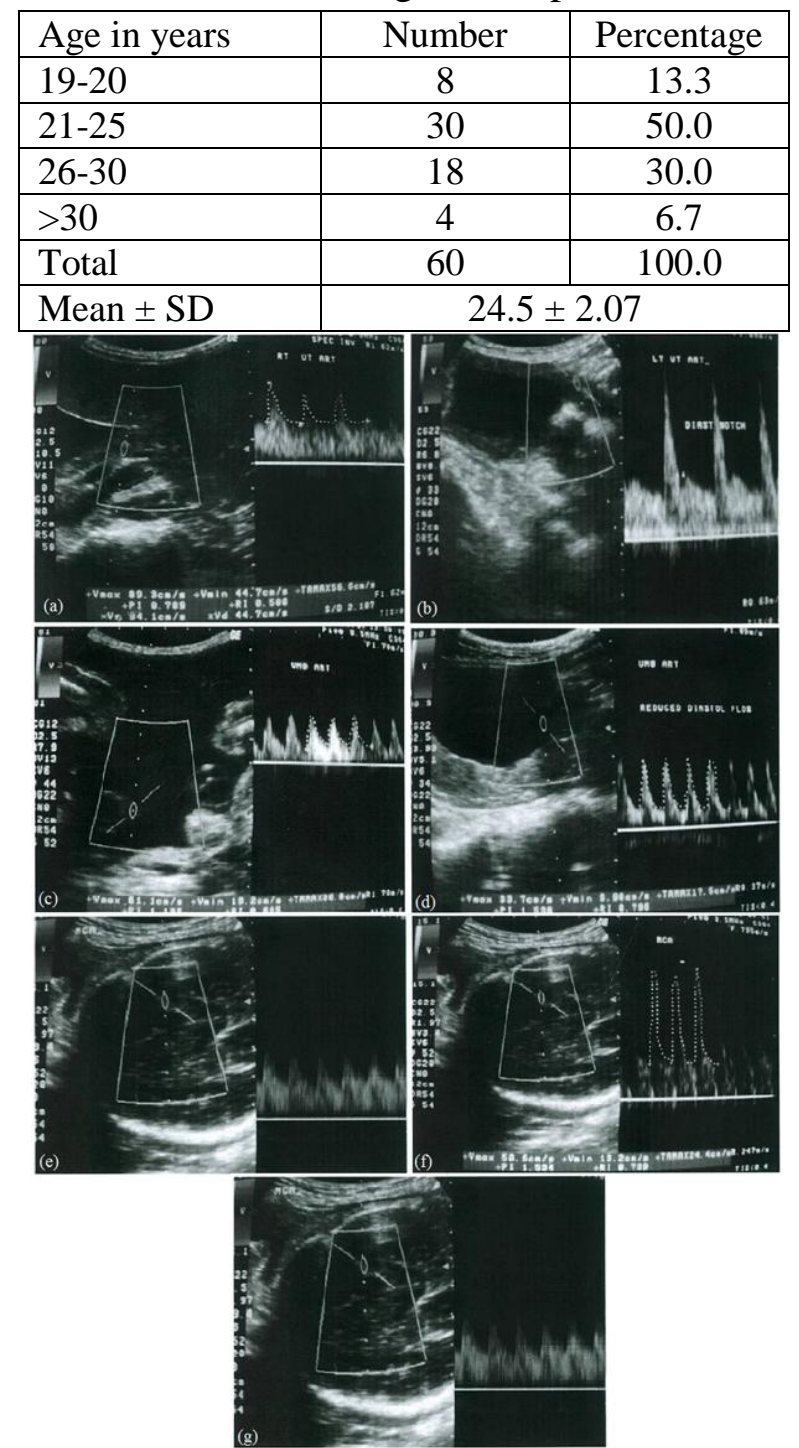

Fig. 1 Typical normal and abnormal waveforms obtained from the uterine (UA), umbilical (UmbA) and middle cerebral arteries (MCA). (a) UA with normal Doppler, (b) UA with early diastolic notch and elevated PI and RI, (c) Umb A with normal Doppler, (d) Umb A with reduced diastolic flow, (e) Umb A with reverse end diastolic flow, (f) MCA with normal Doppler, and (g) MCA with increased diastolic flow and reduced PI.

Distribution of gestational age at Doppler examination

The gestational age of all the study participants at the time of examination ranged between 29-38 weeks. $70 \%$ of the patients were between 31-35 weeks of gestation, $13.3 \%$ were between 26-30 weeks of gestation, and $16.7 \%$ were between $36-40$ weeks of gestation. None of the patients were before 29 weeks of gestation (Table 4).

Table. 4 Distribution of gestational age at Doppler examination

\begin{tabular}{|l|c|c|}
\hline Gestational age & Number & Percentage \\
\hline $26-30$ weeks & 8 & 13.3 \\
\hline $31-35$ weeks & 42 & 70.0 \\
\hline $36-40$ weeks & 10 & 16.7 \\
\hline Total & 60 & 100.0 \\
\hline Mean \pm SD & \multicolumn{2}{|c|}{$33.17 \pm 1.29$} \\
\hline
\end{tabular}

Changes in uterine artery resistance index (RI)

Among 60 patients, 42 (70\%) showed an elevated UA RI and 18 (30\%) showed a normal UA RI. Moreover, out of 42 patients with elevated UA RI, $18(30 \%)$ had an elevated RI in only one uterine artery and 24 (40\%) had bilaterally elevated RI.

\section{Uterine artery early diastolic notch}

Thirty eight (63.3\%) antenatal mothers had a persistent UA early diastolic notch with 16 (26.6\%) had only a unilateral early diastolic notch and $22(36.7 \%)$ had bilateral notches, whereas remaining $22(36.7 \%)$ had normal UA waveform.

Changes in uterine artery using both RI and presence of early diastolic notch

Considering the two parameters, RI and presence of UA persistent early diastolic notch, it has been found that $42(70 \%)$ of the 60 antenatal mothers had an abnormal uterine artery flow velocity profile and $18(30 \%)$ had normal UA flow (Fig. 2).

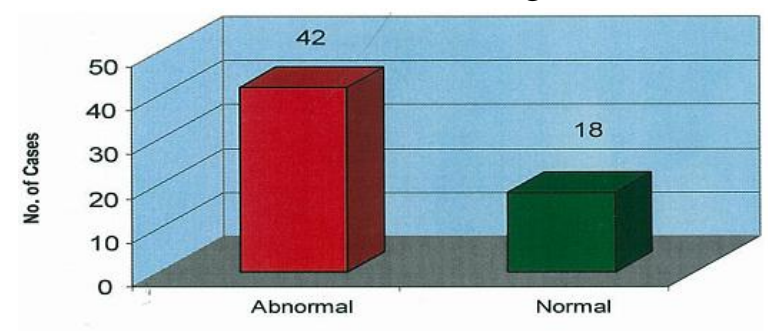

Uterine artery changes

Fig. 2 Doppler findings in the uterine artery using the parameters RI and presence of early diastolic notch. Red bar indicates 42 patients with abnormal UA flow and green bar indicates 18 patients with normal UA flow

\section{Umbilical artery (Umb A) Pulsatility index (PI)}

Umb A PI was detected to be elevated in $36(60 \%)$ patients and normal in 24 (40\%) patients (Fig. 3). 


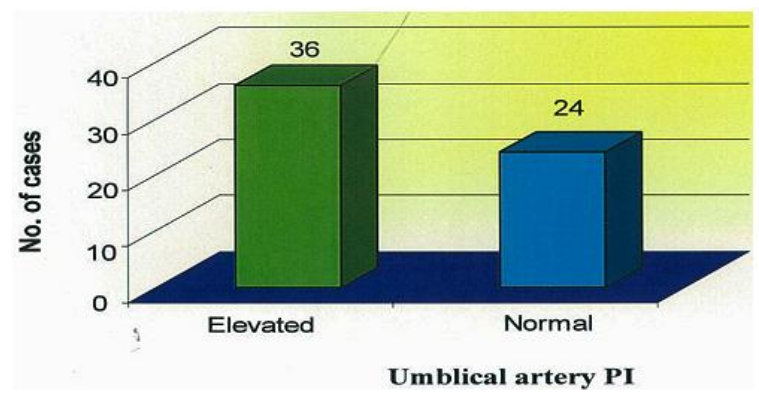

Fig. 3 Distribution of Umb A PI values with gestational age. The green bar indicates 36 patients with elevated Umb A PI and 24 patients with normal Umb A PI

Umbilical artery end diastolic flow velocity pattern

48 (80\%) foetuses showed Umb A positive diastolic flow and 12 showed abnormal waveforms, with ten (16.7\%) had no end diastolic flow, and two (3.33\%) had reverse end diastolic flow (Fig. 4).

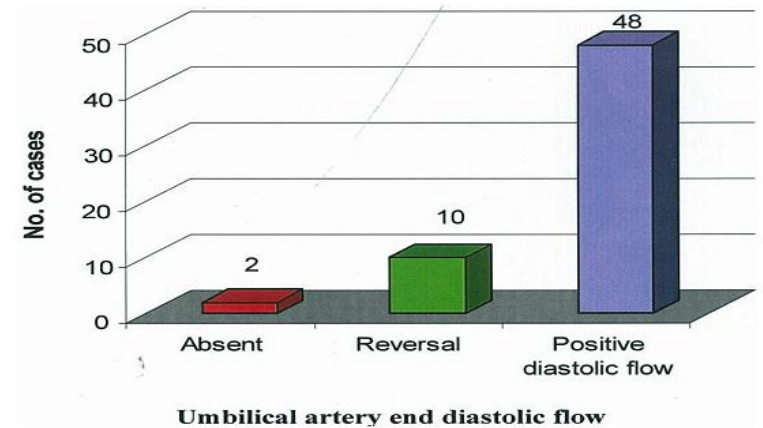

Fig. 4 Doppler velocimetry showing absence or reversal of end diastolic flow in Umb A. Red bar indicates 2 patients with absent end diastolic flow and green bar indicates 10 patients with reversal end diastolic flow. 48 patients had positive diastolic flow as shown by the blue bar

\section{Analysis of both uterine and umbilical arteries}

Doppler evaluation of uterine and umbilical arteries together revealed abnormalities in 48 patients. Conversely, 42 (70\%) of UA and $36(60 \%)$ of Umb A showed irregularities when considered individually (Fig. 5).

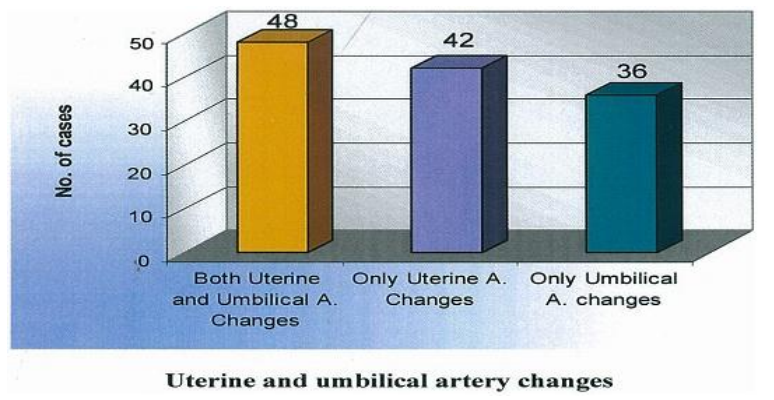

Fig. 5 Doppler evaluation of both uterine and umbilical arteries. Yellow bar indicates abnormalities when both UA and Umb A considered together. Light blue bar indicates only UA changes in 42 patients and deep blue bar indicates only Umb A changes in 36 patients.

Doppler study of foetal middle cerebral artery and determination of foetal blood flow redistribution

Doppler examination of foetal MCA detected a decreased PI in 22 (36.7\%) foetuses and normal PI in $38(63.3 \%)$ foetuses. $63.3 \%$ of foetuses showed redistribution of blood to the brain as evidenced from the MCA/ Umb A PI ratio.

\section{Analysis of perinatal outcome}

Perinatal outcome analysis showed that 38 (63.3\%) foetuses had at least one unfavourable perinatal outcome, whereas $22(36.7 \%)$ foetuses had favourable outcome. There were 54 live births. Adverse results included six intrauterine deaths, admission of 12 neonates to NICU, low APGAR score (less than 7) in 14 neonates, and birth of 18 babies by emergency caesarian section. Mean birth weight at delivery was $2.18 \pm 0.26 \mathrm{~kg}$ (2SD). Forty two neonates had birth weight less than $2.5 \mathrm{~kg}$. Furthermore, of six intra uterine deaths, two cases had absent diastolic flow and one had reverse end diastolic flow. Table 5 showed the comparison of Doppler indices with adverse perinatal outcome. Detailed description of different Doppler indices among study participants is provided in Table 6 (Fig. 6).

Table. 5 Comparison of Doppler indices with adverse perinatal outcome

\begin{tabular}{|l|c|c|c|c|c|}
\hline $\begin{array}{l}\text { Doppler } \\
\text { Index }\end{array}$ & $\begin{array}{c}\text { UA } \\
\text { diast } \\
\text { notch }\end{array}$ & UA R.I & $\begin{array}{c}\text { Umb } \\
\text { A PI }\end{array}$ & $\begin{array}{c}\text { MCA } \\
\text { PI }\end{array}$ & $\begin{array}{c}\text { MCA/ } \\
\text { Umb } \\
\text { PI }\end{array}$ \\
\hline TP & 30 & 28 & 32 & 22 & 34 \\
\hline TN & 16 & 14 & 18 & 20 & 18 \\
\hline FP & 6 & 8 & 4 & 2 & 4 \\
\hline FN & 8 & 10 & 6 & 16 & 4 \\
\hline Sensitivity & $78.9 \%$ & $73.7 \%$ & $84.2 \%$ & $57.8 \%$ & $89.5 \%$ \\
\hline Specificity & $72.7 \%$ & $63.6 \%$ & $81.8 \%$ & $90.9 \%$ & $81.8 \%$ \\
\hline PPV & $83.3 \%$ & $77.8 \%$ & $88.9 \%$ & $91.7 \%$ & $89.5 \%$ \\
\hline NPV & $66.7 \%$ & $58.3 \%$ & $75 \%$ & $55.6 \%$ & $81.8 \%$ \\
\hline $\begin{array}{l}\text { Diagnostic } \\
\text { Accuracy }\end{array}$ & $76.7 \%$ & $70.0 \%$ & $83.3 \%$ & $70.0 \%$ & $86.7 \%$ \\
\hline
\end{tabular}


Table. 6 Performance characteristics of different Doppler indices

\begin{tabular}{|l|c|c|}
\hline Doppler indices & Number & Percentage \\
\hline 1. Elevated UA RI & 42 & 70.0 \\
\hline 2. UA early diastolic notch & 38 & 63.3 \\
\hline 3. Both UA parameters & 42 & 70.0 \\
\hline 4. Elevated Umb A PI & 36 & 60.0 \\
\hline 5. UA and Umb A & 48 & 80.0 \\
\hline 6. Abnormal MCA PI & 22 & 36.7 \\
\hline 7. MCA/Umb A PI & 38 & 63.3 \\
\hline
\end{tabular}

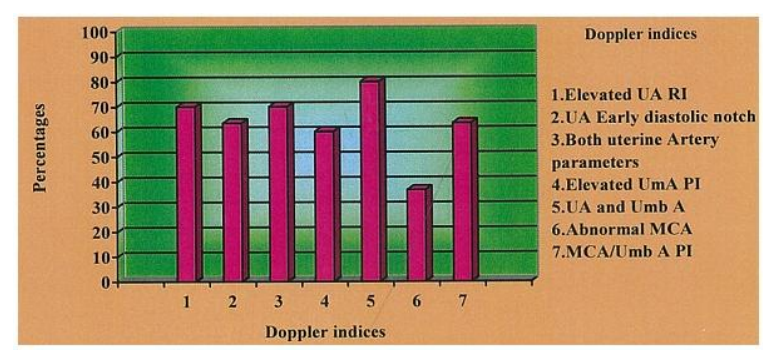

Fig. 6 Performance characteristics of Doppler indices. 7 different Doppler indices were analysed. UA and Umb A Doppler indices showed highest performance (around 80\%) and abnormal MCA showed lowest performance (around 35\%).

\section{DISCUSSION}

The early prediction of adverse perinatal outcomes in IUGR allows the initiation of management strategies that may inhibit or mitigate these complications. Accurate prenatal diagnosis determines whether the foetus is constitutionally small or small due to damaged placental perfusion. UA and Umb A Doppler velocimetry studies enable the assessment of uteroplacental and foeto-placental unit, and also foetal adaptation towards changes in intra uterine environment. Increased abnormality in Doppler images indicates higher fetal compromise [10].

Numerous studies describing the pregnancy complications due to IUGR with varying results have been published. This discrepancy might arise from varying sample sizes, small number of patient enrollment, inadequate understanding of IUGR pathophysiology, use of diverse techniques, as well as different criteria to define adverse perinatal outcome. Due to the lack of universal guidelines to monitor growth of IUGR foetus, conflicting observations might continue to evolve. Consequently, the decision to deliver preterm IUGR foetus becomes one of the biggest challenges in obstetrics $^{[6]}$.

In the present study including 60 pregnant women, the maximum number of participants belonged to the age group of 21-25 years, probably due to the increased rate of pregnancy in this age group. All the patients at the third trimester of their pregnancy underwent Doppler study. 70\% was investigated between 31 - 35 weeks of gestation and the earliest study was done at 291/2 week of gestation. The gestation period of 31-35 weeks had been selected because during this period the foetus normally begins to develop sufficient lung maturity to survive outside the uterus.

Among the study participants, $56.67 \%$ was detected with pregnancy induced hypertension $(\mathrm{PIH}), 16.67 \%$ with severe anemia. Hypertensive disorders were present in $30 \%$ to $40 \%$ of pregnancies, $6.67 \%$ had poor obstetric history, and $26.67 \%$ had no detectable cause for IUGR.

UA Doppler Velocimetry is widely used to predict pregnancy outcome. UA early diastolic notch depicts the reflected blood flow of uteroplacental circulation ${ }^{[16]}$. The present study detected the sensitivity of UA diastolic notch beyond 26 weeks of gestation to be $78.9 \%$. This value is slightly higher than the findings by Coleman et al [17] (sensitivity $76 \%$ for adverse perinatal outcome) and lower than the findings by Farrell et al [18] (sensitivity 88\%). This difference in value is probably because of the variation in study population since unilateral diastolic notches were considered in this study in contrast to bilateral notches by Coleman et al ${ }^{[17]}$. The sensitivity of UA RI was found to be $73.7 \%$, in comparison to $67 \%$ detected by Benson and Doubliet ${ }^{[19]}$ and $83 \%$ by Coleman et al ${ }^{[17]}$. This discrepancy might result from different cut of values of RI, ranging between $0.5-0.62$.

When both Doppler parameters (RI and UA early diastolic notch) were taken into account, the sensitivity for predicting critical consequence was $83 \%$. This is in agreement to the study by Zimmermann et al ${ }^{[20]}$ who found a combination of several Doppler parameters to be superior to a single parameter. 
Umbilical artery is considered to be the principal vessel for monitoring high risk pregnancies since, it represents foetoplacental system and primarily reflects placental resistance to blood flow. With pregnancy progression, a low resistance in the Umb A results in a good diastolic flow, whereas, a high resistance in the Umb A results in absence/reversal of diastolic flow. Our study detected the sensitivity of Umb A PI in predicting perinatal outcome to be $84.2 \%$. According to the studies by Gramellini et al ${ }^{[13]}$ the sensitivity of Umb A PI was $64 \%$ and by Fong et al ${ }^{[21]}$ the sensitivity of Umb A PI was $58.3 \%$. The presence of increased number of $\mathrm{PIH}$ could attribute to this difference. Absence or reversal of the end diastolic flow velocity was seen in $20 \%$ fetuses in contrast to $37 \%$ detected by Benson and Doubilet ${ }^{[19]}$. The MCA Doppler is easy to perform compared to the UA and Umb A Doppler, since the vessel lies in the plane of the ultrasound beam, resulting in strong color signal ${ }^{[22]}$. Foetal MCA PI aids in the assessment and monitoring of foetal oxygenation. In the present study, $37 \%$ of foetuses showed a decrease in MCA PI and the sensitivity of foetal MCA PI was $57.8 \%$. Arduini and Rizzo ${ }^{[23]}$ detected the sensitivity of MCA PI as $68 \%$ in predicting perinatal outcome. However, the current study cannot be compared with the study by Arduini and Rizzo ${ }^{[23]}$ due to the difference in consideration of the intra cranial artery, and it has been clearly established that PI varies in relation to the intra cranial artery. Hence, accurate identification of the specific artery is vital in assessing fetal outcome. $63 \%$ participants showed redistribution of blood to the brain when calculated using MCA PI/ Umb A PI while the redistribution calculated usingUmb A PI and MCA PI alone were $60 \%$ and $36.7 \%$ respectively. The sensitivity of MCA PI / Umb A PI in predicting perinatal outcome was $89.5 \%$. Actually, MCA/Umb A PI ratio had a higher sensitivity and positive predictive value in predicting adverse perinatal outcome than MCA and Umb A PI separately. The finding of our study is in conformity with that of Gramellini et al [13]. However, use of uniform or standardized criteria is necessary to compare the results of different studies.
$63 \%$ foetuses showed at least one adverse outcome. There were 54 live births and six intra uterine deaths. Four intrauterine death cases had absent diastolic flow and one had reversal of diastolic flow. Of the 54 live births, 12 neonates were admitted to NICU, four had 5 min APGAR score less than 7, and 18 neonates were delivered by emergency caesarian section. These results are slightly higher than that of Gramellini et al ${ }^{[13]}$ and might be due to the variation in perinatal mortality and morbidity rates between western and Indian standards.

\section{CONCLUSION}

In conclusion, the current study confirmed the superiority of cerebroumbilical ratio over the MCA PI or Umb A PI alone in the detection of early onset of IUGR and adverse perinatal outcome. The umbilical-placental and cerebral vascular beds are directly involved in the hemodynamic adjustments of foetal growth restriction. A Doppler index that reflects both of these areas can be useful for identifying foetuses with increased placental and/or decreased cerebral resistance.

Furthermore, the present study focused on the importance of combined assessment of the uteroplacental and the foetoplacental circulations in estimation of unfavourable perinatal outcome. UA early diastolic notch has been believed to be a useful parameter to monitor uteroplacental circulation. Additionally, the present study indicated the absent/reversal of end diastolic flow in Umb A as the best predictor of poor foetal consequences such as chronic hypoxia, growth retardation, and high mortality.

Colour Doppler imaging aids in better understanding of foetal hemodynamics. Hence, it is beneficial in monitoring the pregnancy, predicting adverse foetal outcome, and timely delivery of IUGR foetuses, which in turn can reduce foetal morbidity and mortality. However, further studies are required to analyze the role of colour Doppler velocimetry to optimize foetal outcome.

\section{REFERENCES}

1. Berkley E, Chauhan SP., Abuhamad A. Doppler assessment of the fetus with 
intrauterine growth restriction. Am J Obstet Gynecol 2012: 206: 300-308. doi: 10.1016/j.ajog.2012.01.022

2. Galbraith RS, Kershmar EJ, Peircy WN, Low JA. The clinical prediction of intrauterine growth retardation. Am J Obstet Gynecol 1979: 133: 281-286. doi: 10.1016/0002-9378(79)90679-3

3. Sharma D, Shastri S, Sharma P. Intrauterine Growth Restriction: Antenatal and Postnatal Aspects. Clin Med Insights Pediatr 2016: 10: 67-83. doi: 10.4137/CMPed.S40070

4. Suhag A, Berghella V. Intrauterine Growth Restriction (IUGR): Etiology and Diagnosis. Curr Obstet Gynecol Rep 2013: 2: 102-111. doi:10.1007/s13669-013-0041-Z

5. Jain S, Puri M. Diagnosis of intrauterine growth retardation - A review. Obstet and Gynec Today 2001: 11: 670-673.

6. Giuliano N, Annunziata ML, Tagliaferri S. et al. IUGR Management: New perspectives. J Pregnancy. 2014: 620976: 8. doi: http://dx.doi.org/10.1155/2014/620976

7. Ghosh GS, Gudmundsson S. Uterine and umblical artery Doppler are comparable in predicting perinatal outcome of growth restricted fetuses. BJOG 2009;116:424 - 430.

8. Mone F, McAuliffe FM, Ong S. The clinical application of Doppler ultrasound in obstetrics. Obstet Gynecol. 2015; 17: 13-19.

9. Kok JH, Devi Ouden AL, VerlooveVenhorick SP, Brand R. Outcome of very preterm small for gestational age infants : the first nine years of life. Br J Obstet and Genecol 1989: 105: 162-168.

10. Savithri DR, Pallavi R, Reethu Varadarajan, Veena BT. Optimizing perintala outcome in high risk pregnancies using Doppler velocimetry. JEMDS 2015: 4: 16064-16068. doi: 10.14260/jemds/2015/2346

11. Krishna U, Bhalero S. Placental insufficiency and fetal growth restriction. J Obstet Gynaecol India 2011: 61: 505-511. doi: 10.1007/s13224-011-0092-x

12. Hemlata D, Kumar KH, Anupama D. Middle Cerebral Artery Doppler Indices
Better Predictor for Fetal Outcome in IUGR. J Obstet Gynaecol India 2011: 61: 166-171. doi: 10.1007/s13224-011-0018-7

13. Gramellini D, Folli MC, Raboni S, Vadora E, Merialdi A. Cerebral-umbilical Doppler ratio as a, predictor of adverse perinatal outcome. Obstet Gynecol 1992: 79: 416-420.

14. Wladimiroff JW, Wijingaard JAGW, Degani S, Noordam MJ, van Eyck J, Tonge HM. Cerebral and umbilical arterial blood flow velocity waveforms in normal and growth retarded pregnancies. Obstet Gynecol 1987: 69: 705- 709.

15. Lugo G, Cassady G. Intrauterine growth retardation clinico pathological findings in 233 conseptive infants. Am J Obstet Gynecol 1971:109: 615- 122.

16. Park YW, Cho Js, Choi HM, Kim TY, Lee $\mathrm{SH}, \mathrm{Yu}$ JK, Kim JW. Clinical significance of early diastolic notch depth: uterine artery Doppler velocimetry in the third trimester. Am J Obstet Gynecol 2000: 182: 1204-1209.

17. Coleman MA, McCowan LM, North RA. Mid-trimester uterine artery Doppler screening as a predictor of adverse pregnancy outcome in high-risk women. Ultrasound Obstet Gynecol 2000: 15: 4-6. doi: 10.1046/j.1469-0705.2000.00014.x

18. Farrell T, Chien PF, Mires GJ. The reliability of the detection of an early diastolic notch with uterine artery Doppler velocimetry. $\mathrm{Br}$ J Obstet Gynaecol 1998: 105: 1308-1311.

19. Benson CB, Doubilet PM. Doppler criteria for intrauterine growth retardation: predictive values. J Ultrasound Med 1988: 7: 655-659. doi: 10.7863/jum.1988.7.12.655

20. Zimmermann P, Eirio V, Koskinen J, Kujansuu E, Ranta T. Doppler assessment of the uterine and uteroplacental circulation in the second trimester in pregnancies at high risk for pre-eclampsia and/or intrauterine growth retardation: Comparison and correlation between different Doppler parameters. Ultrasound Obstet Gynecol 
1997: 9: 162-172. doi: 10.1046/j.14690705.1997.09050330.x

21. Fong KW, Ohlsson A, Hannah ME, Grisaru S, Kingdom J, Ryan M, Windrim R, Foster G, Amankwah K.. Prediction of Perinatal Outcome in Fetuses Suspected to have Intrauterine Growth Restriction: Doppler US Study of Fetal Cerebral, Renal and Umbilical Arteries. Radiology 1999: 213: 681-689. doi:

10.1148/radiology.213.3.r99dc08681

22. Hershkovitz R, Kingdom JCP, Geary M, Rodeck CH. Fetal cerebral blood flow redistribution in late gestation: identification of compromise in small fetuses with normal umbilical artery Doppler. Ultrasound Obstet Gynecol 2000: 15: 209-212. doi: 10.1046/j.1469-0705.2000.00079.x

23. Arduini D, Rizzo G, Romanini C, Mancuso S. Fetal blood flow velocity waveforms as predictors of growth retardation. Obstet Gynecol 1987: 70: 7-10. 